\title{
A New Joining Algorithm for Multi-hop Non-mobile Wireless Sensor Networks
}

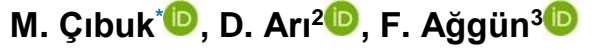 \\ ${ }^{1 *}$ Bitlis Eren University, Department of Computer Engineering, Bitlis, Turkey, (mcibuk@beu.edu.tr) \\ 2 Bitlis Eren University, Department of Computer Engineering, Bitlis, Turkey, (dari@beu.edu.tr) \\ ${ }^{3}$ Bitlis Eren University, Department of Computer Technologies, Bitlis, Turkey, (faggun@beu.edu.tr)
}

\section{ARTICLE INFO}

Received: Apr., 15. 2021

Revised: Sep., 24. 2021

Accepted: Sep., 29. 2021

Keywords:

Wireless Sensor Networks

Relay Mechanism

Channel Allocation

Joining Algorithm

Corresponding author: $M$. Çıbuk

ISSN: 2536-5010 | e-ISSN: 2536-5134

DOI: https://doi.org/10.36222/ejt.916717

\section{ABSTRACT}

Wireless Sensor Networks (WSN) are divided into two categories as single-hop and multihop according to the connection states of the sensor nodes. In the single-hop WSNs, the sensor nodes communicate directly with the management unit (coordinator node). On the other hand, in multi-hop WSNs, the sensor nodes, which are out of the coverage area of the coordinator node communicate with the coordinator over other joined sensor nodes. Multihop WSNs preferred for complex applications where there are many sensor nodes. In such applications, connecting to the network and maintaining the continuity in the network is difficult and requires very complicated algorithms for sensor nodes.

In this study, a new network-joining algorithm for multi-hop WSNs has been proposed and designed. According to the algorithm, there is a management unit which is named as coordinator node $(\mathrm{CN})$ for maintaining the network connectivity and the nodes which are the members of the network sends network joining requests over a common contentionbased channel for joining the network. If there is a coordinator node in the coverage area, the sensor node joins directly to the network and coordinator allocates an appropriate channel to the new node. If the channel has multiple users, it is used as time-shared. If the sensor node is out of coverage area, directly joining to the network is impossible. In this case, the sensor node sends a relay request to the other joined sensor nodes. The available sensor node responds to this relay request via handshake. The sensor node uses the relay node's channels as shared by using time-sharing methods. Therefore, the new node joins the network from the relay node's channel. The main goal of this algorithm is to speed up joining of sensor nodes to the networks in multi-hop WSNs. Also, another goal is maximizing the continuity of the sensor nodes in the network. Thus, the algorithm improves performance and ensures data transfer continuity of WSNs.

\section{INTRODUCTION}

Wireless Sensor Networks (WSNs) consists of Sensor Nodes (SNs) connected wirelessly with each other. In the WSNs, there are sensor nodes and a coordinator node as basic network elements. The Coordinator Node $(\mathrm{CN})$ organizes the other network elements. The most basic feature of sensor nodes is to sense environmental data, to process sensed data and to transmit processed data[1]-[3]. The required sensor node count changes from application to application. For example, in order to track environmental imaging and natural catastrophes, because of the land structure, requires a large number of sensor nodes. It is obvious that increasing the number of sensor nodes in the network makes more complicated the network. Complex structured WSNs use a multi-hop network structure as a connection method. In such networks, as shown in Figure 1, the sensor nodes extend coverage of the network by communicating with each other [4], [5]. In multi-hop networks, the sensor nodes perform not only their own sensing tasks but also for its neighbor nodes the relay function. Multi-hop WSNs have advantages such as coverage, high data transmission rate, low cost[6]. Thanks to these networks, which spread over large areas, data can be collected by consuming less energy. Thus, the lifetime of the network extends[7].

On the contrary of the above mention advantages of the multi-hop WSN, there are some difficulties. Difficulties which foreseen in the multi-hop WSN design are negative factors such as congestion[1], end-to-end delay, hidden node, simultaneous communication, a disconnection of nodes from the network, topology changes[4], and fairness[6]. These difficulties have a negative impact on the performance of the network. When looking at the literature[8]-[11], the existence of studies can be observed on which contains the solution of these difficulties. While the studies, which do for the postsetup phase of the network are more frequent, the studies for network initialization phase seems to be less. 


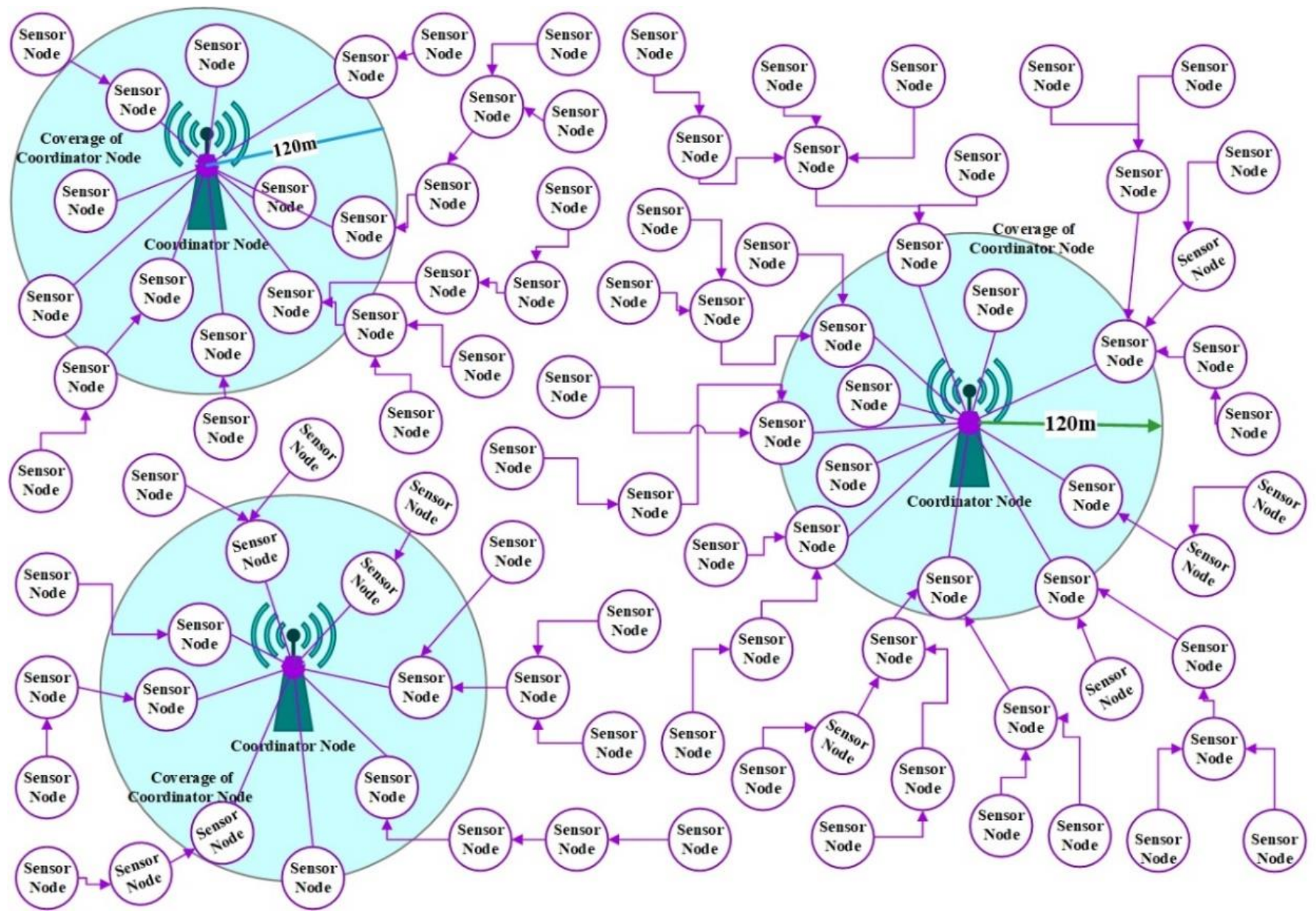

Figure 1. Connection state of Multi-hop Wireless Sensor Networks

In this study, a new joining network algorithm have been developed for the multi-hop non-mobile WSNs. According to the algorithm, the sensor nodes have sensing functions as well as a relay function that for other nodes can be used when needed. Furthermore, in this algorithm, a multi-channel (FDMA) structure has been used to minimize the collision. The nodes use a common contention-based (CSMA-CA) channel for their request to join the network, after which for the communication the nodes uses the channel which allocated by the $\mathrm{CN}$. The sensor node directly joins the network if it is in the coverage area of the $\mathrm{CN}$. If the sensor node is not in the coverage area of $\mathrm{CN}$, the nodes send a request in order to join the network to the nearest sensor node, which has joined before. Neighbor sensor node, which received this networkjoining request, sends a request to the $\mathrm{CN}$. If $\mathrm{CN}$ accepts to request, the node sets the request-sender node as its relay node and use channels of the relay node. The nodes communicate with the $\mathrm{CN}$ as time-shared (TDMA) by using relay node channels. The performance of this algorithm has been tested based on node counts at random form of nodes in the Riverbed Modeler simulation environment.

\section{A NEW JOINING ALGORITHM FOR MULTI-HOP NON-MOBILE WIRELESS SENSOR NETWORKS}

According to the algorithm, firstly, the nodes listen to the CH_REQUEST channel and attempts to obtain the CH_REQUEST channel by using the CSMA-CA method, as shown in Figure 2. CH_REQUEST is a common channel, which nodes use to send CTRL_PKT (control packet, network-join request) packets to the $\mathrm{CN}$. When the node obtained the channel, it sends a CTRL_PKT to the CN. Later, the node starts to listen to the CH_SCHEDULE channel that $\mathrm{CN}$ uses to send the SCH_PKTs which tell conditions of nodes in the network to the SNs. After this stage, the node starts a counter to hold the Count for not scheduled and a wait_time counter for determining the time without getting any packet. If there is $S C H \_P K T$ on the channel and a channel allocation is present for itself, the node will be connected to the CN directly. Otherwise, if there is $S C H \_P K T$ on the channel but channel allocation is not for itself counter will be incremented and it will be returned to listen to CH_SCHEDULE channel state until the counter exceeds the threshold_count threshold. If there is not any packet on the CH_SCHEDULE channel within the wait_time the node sets CTRL_PKTs relay/request bit as 1 and starts to broadcast a this packet to the neighboring sensor nodes.

The neighboring node that received this packet will send a RRQUEST_PKT (relay request packets which supply to join as a relay to the network) to the $\mathrm{CN}$. The relay node becomes the parent of the sensor node, which wants to join the network. After this stage, the relay node starts to send the $S C H \_P K T$ s which coming from $\mathrm{CN}$ to all child nodes by using the $\mathrm{CH}_{-}$SCHEDULE channel. The searching relay node or connecting $\mathrm{CN}$ directly period continues as described below. After this step, the node repeats the same phase as the phase of direct $\mathrm{CN}$ connection that mentions above, as shown in Figure 2. 


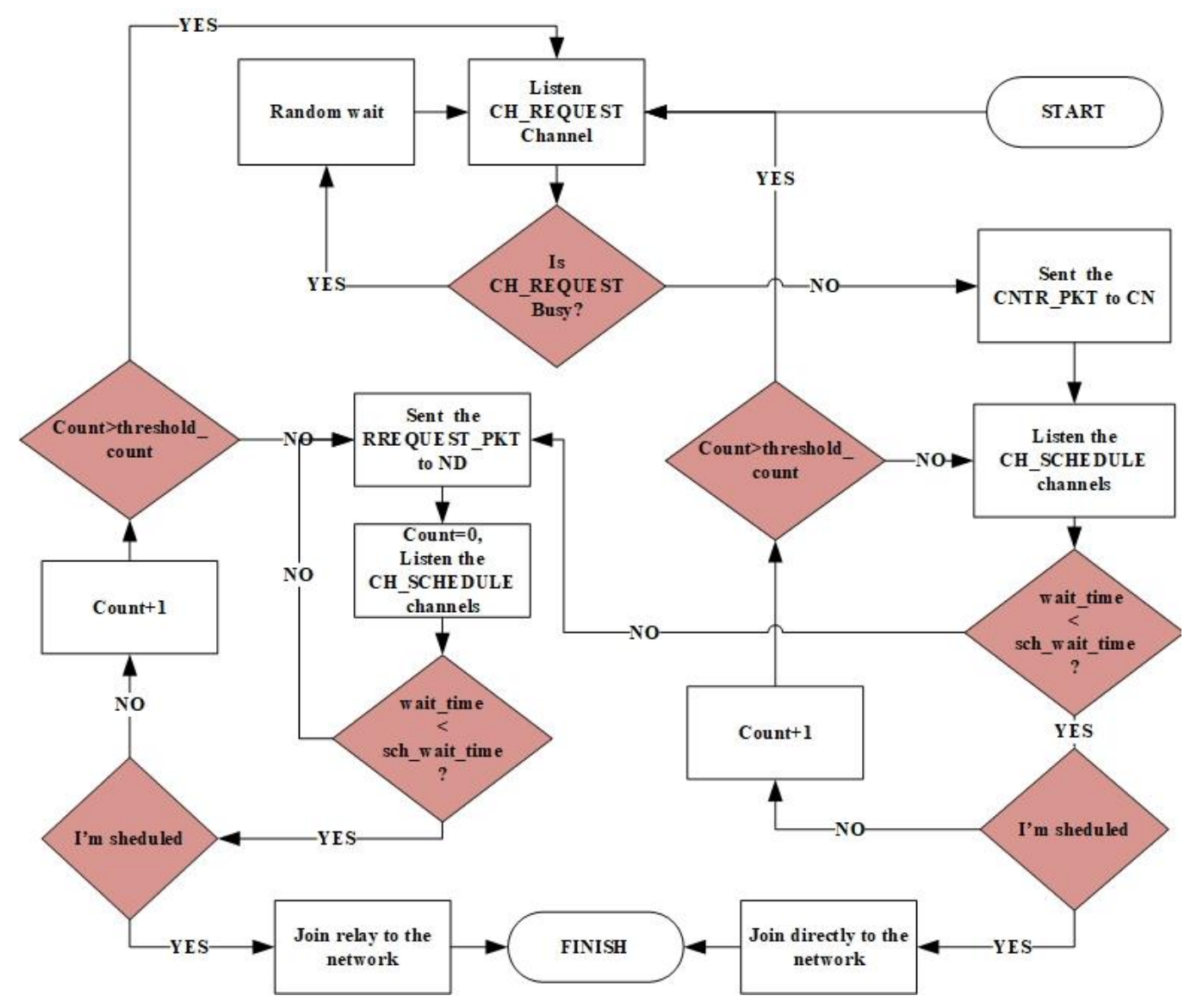

Figure 2. Flowchart of the algorithm

Start

Initial_Values_Set, Count=0, Time_out, wait time

RRQUESTTPKT, SCH_PKT, CTRL_PKT,

$\mathrm{CH}$ SCHEDUUL, CH $\overline{\mathrm{R} E Q U E S T}, \mathrm{C} \overline{\mathrm{N}}$, ND;

Stēp: 1 Listen CH_REQUEST channel

Step:2 If (Is $\mathrm{CH}^{-}$REQUEST channel busy?)

Random wait;

GoTo Step1;

Else

Sent CTRL_PKT to CN

GoTo Step $\overline{4}$;

Step: 3 While (Listen $\mathrm{CH}_{-}$SCHEDULE channel) \{

If (Is there SCH_PKT)

Else GoTo Step4;

$$
\text { GoTo Step5 }
$$

Step: 4 If (I'm scheduled)

$$
\text { GoTo Step7; }
$$

Else

Count++;

If (Count $=>$ threshold_count)

GoTo Step5;

Else

GoTo Step1;
\}

Step: 5 sets CTRL_PKTs relay/request bit TRUE Send CTRL_PKT

Count $=0$;

While (Listen $\mathrm{CH}$ SCHEDULE channel) \{ If (Is there SCH_PKT)

Else GoTo Step $\overline{6}$;

GoTo Step5

Step: 6 If (I'm scheduled) GoTo Step7;

Else

Count++;

If (Count $=>$ threshold_count)

GoTo Stepl;

Else

GoTo Step5;

\}

Step: 7 I'm joining the network

Finish; 


\section{SIMULATION AND TEST}

In this study, Riverbed (Opnet) [12] Modeler simulation environment has been preferred because it has many advantages such as an advanced graphical interface, hierarchical modeling, and simultaneously simulations with multiple inputs to test the algorithm. It also supports from very small networks to very large networks [13].

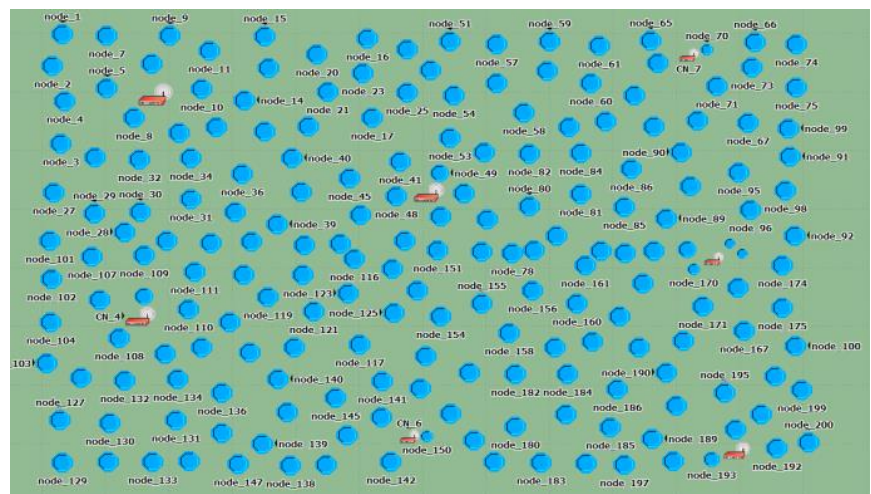

Figure 3. Scenario of 200 node in Riverbed Modeler network project environment $(3200 \mathrm{mX} 3200 \mathrm{~m})$

In this study, the node counts have been defined as 25,50 , $100,200,400,600,800,1000$ to test the effect of node counts on network joining. The maximum coverage area of both $\mathrm{CNs}$ and $\mathrm{SNs}$ is set to $120 \mathrm{~m}$. Both CNs and nodes are randomly distributed in the simulation area. Thus, a multi-hop structure has been achieved. There are seven CNs in the network and each uses a different schedule channel. In order for the algorithm to have a flexible structure, the SNs configured to listen to all the $\mathrm{CN}$ channels. Network environments have been shown in Figure 3.

\section{CONCLUSION}

The proposed algorithm has shown that all nodes in all scenarios join to the network. As the number of nodes in the scenario increases, hop count increases, so the network joining latency have increased. In Figure 4, it has been shown that status of the node joining to the network for SN side in all scenarios. In Figure 5, it has been shown that status of the node joining to the network for $\mathrm{CN}$ side in all scenarios. As seen from the figures the differences between graphs are very small. As the number of nodes increases, the fluctuations in joining network have increased. Even if there is a delay as seen in the figures, all the nodes have joined the network.

Our main goal is not to join the nodes to the $\mathrm{CN}$ neither early nor late, but all nodes connect directly or indirectly (relay) to the $\mathrm{CN}$.

Another goal of developed algorithm in this study is to ensure the continuity of connected nodes. It is obvious that the fast reconnection of nodes that are disconnected from the network is very important for the performance of the network. Looking at the results, it is seen that the proposed algorithm produces realistic results close to one hundred percent in network joining.

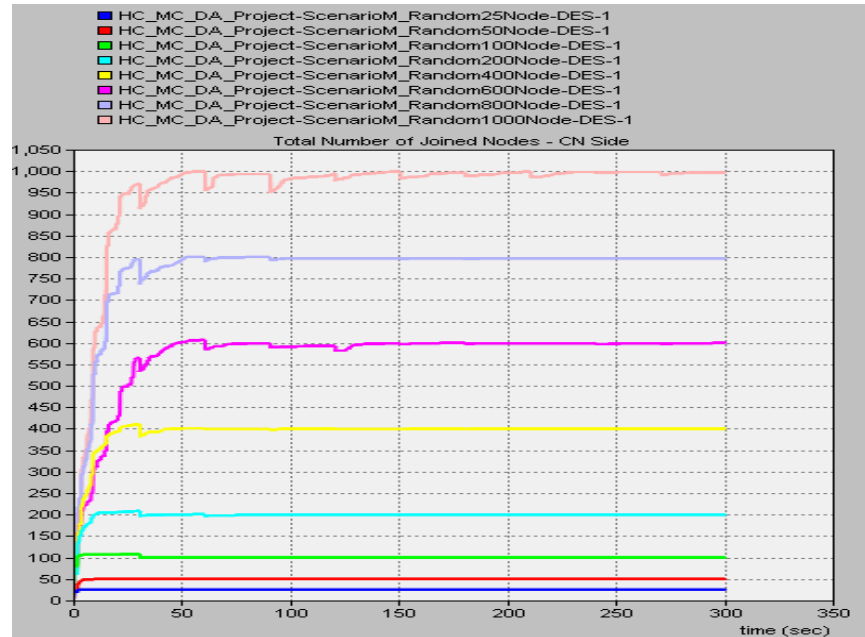

Figure 4. Total Number of Joined Nodes - ND Side

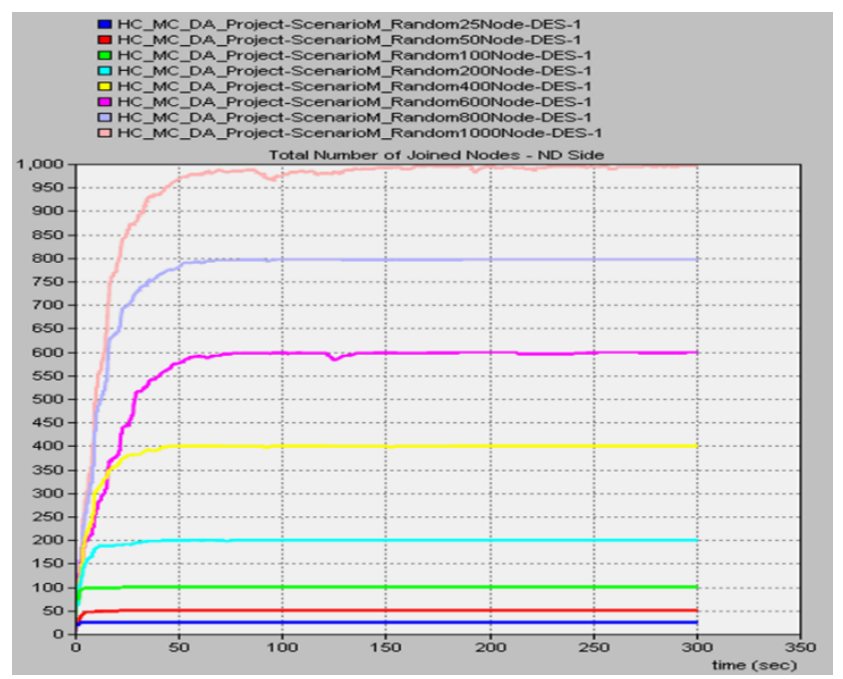

Figure 5. Total Number of Joined Nodes - CN Side

In this study, network joining algorithm considered for the non-mobile WSN networks and performance analysis has performed in the simulation environment. In the future, it is planned to adapt this algorithm for WSN networks consisting of mobile sensor nodes.

\section{ACKNOWLEDGEMENT}

The only abstract of this paper was presented in ICCESEN'17 conference. This paper is an extended full text version of previously presented abstract in ICCESEN'17.

\section{REFERENCES}

[1] O. Chughtai, N. Badruddin, and A. Awang, 'A novel congestion alleviation procedure in multi-hop wireless sensor networks', 2016 6th International Conference on Intelligent and Advanced Systems (ICIAS), IEEE,pp. 1-6, 2016.

[2] F. Alshahrany, M. Abbod, and I. Moualek, 'WSN and RFID Integration to Support Intelligent Monitoring in Smart Buildings Using Hybrid Intelligent Decision Support Systems', Acta Phys. Pol. A, vol. 128, no. 2B, ,p. B-152-B-160, 2015.

[3] C. Z. Zulkifli, H. N. Hassan, W. Ismail, and S. N. Semunab, 'Embedded RFID and Wireless Mesh Sensor Network Materializing Automated Production Line Monitoring', Acta Phys. Pol. A, vol. 128, no. 2B, ,p. B-86-B-90, 2015. 
[4] P. Murdiyat, K. S. Chung, and K. S. Chan, 'A multi-channel MAC for multi-hop wireless sensor networks minimizing hidden node collision', 2016 22nd Asia-Pacific Conference on Communications (APCC), IEEE,pp. 535-540, 2016.

[5] P. Murdiyat, K. S. Chung, and K. S. Chan, 'Predicting the network throughput of wide area WSN in rural areas', The 20th Asia-Pacific Conference on Communication (APCC2014), IEEE,pp. 106-111, 2014.

[6] D. D. Vergados, D. J. Vergados, A. Sgora, D. Vouyioukas, and I. Anagnostopoulos, 'Enhancing Fairness in Wireless Multi-Hop Networks', Proceedings of the 3rd International ICST Conference on Mobile Multimedia Communications, ICST,pp. 1-6, 2007.

[7] C. Duan, F. Shi, X. Ding, X. Xiao, and P. Duan, 'A novel TDMA and multi-hop MAC protocol in cluster-based wireless sensor networks', 2011 6th IEEE Conference on Industrial Electronics and Applications, Bitlis,IEEE,pp. 805-808, 2011.

[8] K. Nguyen and Y. Ji, 'Achieving Minimum Latency in Multi-Hop MAC Protocol for Wireless Sensor Networks', 2011 IEEE 73rd Vehicular Technology Conference (VTC Spring), IEEE,pp. 1-5, 2011.

[9] Ji-Jun Zhao and Xiang Sun, 'MAC protocol based on T-MAC multihop reservation for short-latency wireless sensor network', 2008 11th IEEE International Conference on Communication Technology, Hangzhou, China,IEEE,pp. 114-117, 2008.

[10] K. Nguyen and Y. Ji, 'AM-MAC: an energy efficient, Adaptive Multihop MAC protocol for sensor networks', Proceedings of the 6th International Wireless Communications and Mobile Computing Conference on ZZZ - IWCMC '10, New York, USA,ACM Press,p. 432, 2010.

[11] J.-W. Lee and H.-S. Cho, 'Cascading Multi-Hop Reservation and Transmission in Underwater Acoustic Sensor Networks', Sensors, vol. 14, no. 10, ,pp. 18390-18409, 2014.

[12] Riverbed Modeler, 'Riverbed Modeler', https://www.riverbed.com/gb/products/steelcentral/steelcentralriverbed-modeler.html,accessed 9 April 2021.
[13] X. Chang, 'Network simulations with OPNET', Proceedings of the 31 st conference on Winter simulation Simulation-a bridge to the future - WSC '99, vol. 1, New York, USA,ACM Press,pp. 307-314, 1999.

\section{BIOGRAPHIES}

Musa Çıbuk obtained his bachelors' degree in the Electrical-Electronic Engineering Department at Firat University, Elazığ/Turkey in 1997, his master degree in the Electrical-Electronic Engineering Department at Firat University, Elazı $\breve{g} /$ Turkey in 2002 and $\mathrm{PhD}$ degree in the ElectricalElectronic Engineering Department at Firat University, Elazığ/Turkey in 2009. He is still an academic member of the Computer Engineering Department at Bitlis Eren University. His major areas of interests are: Wireless Communication, Internet, Server Systems, Sensor Networks and Image Processing.

Davut Arı obtained his BSc degree in computer engineering from Harran University (HRU) in 2014. He received the MSc diploma in Electric Engineering from the Bitlis Eren University in 2018. Her research interests are Computational Intelligence, Genetic Programming, Wireless Sensor Networks. In 2015 he joined the Computer Enginering, Faculty of Engineering And Architecture, Bitlis Eren University as a research assistant.

Fikri Ağgün obtained his bachelors' degree in the Computer Engineering Department at Selçuk University, Konya/Turkey in 2001, his master degree in the Biometry and Genetic Department at Yüzüncü Y1l University, Van/Turkey in 2011, Phd Study is resuming. He is still an academic member of the Informatics Department at Bitlis Eren University. His major areas of interests are: Computer Networks, Wireless Sensor Networks and Vehicular Adhoc Networks. 